\title{
Clinical presentations of vascular malformations of the brain stem: comparison of angiographically positive and negative types
}

\author{
MASAMITSU ABE, RAYMOND N KJELLBERG, RAYMOND D ADAMS \\ From the Departments of Neurosurgery and Neurology, Massachusetts General Hospital, Boston, MA, USA
}

SUMMARY Clinical and radiographic features of 63 patients with a vascular malformation of the brain stem are described. On radiological grounds they were divided into two groups: one with angiographically visible lesions (AVAVMs), the other with lesions not seen angiographically, that is, occult (AOVMs). In the first group the initial clinical manifestation was due to haemorrhage in 20 of the 33 cases and consisted of a progressive neurological deficit in 12. In the second group 29 of the 30 initially presented with a brain stem haemorrhage. The latter was often characterised by development of symptoms over two days or more (16 cases), absence of headache (48 cases) and tendency to recurrence (20 cases). Clinical diagnosis was difficult in many cases especially in the AOVM group. Several of the patients were misdiagnosed as having multiple sclerosis. Clinical data in conjunction with magnetic resonance imaging were helpful in determining the nature of these lesions.

While treating some 900 patients with cerebrovascular malformations unsuitable for surgical removal, we encountered a considerable number of patients with a vascular malformation of the brain stem, many of which were not revealed by angiography. We agree that diagnosis of angiographically occult vascular malformations (AOVMs) is now possible by using the new methods of imaging, particularly magnetic resonance. ${ }^{1-3}$ Nevertheless, even that technique does not eliminate difficulties in diagnosis of some AOVMs because other types of lesion (that is, tumours and bleeding disorders) may give a similar picture. Once diagnosis is made there is still controversy over the need for treatment of brain stem AOVMs ${ }^{47}$ because their natural history has not been sufficiently studied. Our main objectives in undertaking this study were to establish certain principles of diagnosis and to provide additional data concerning the natural history of cerebrovascular malformations.

\section{Subjects and methods}

The first 63 of our patients with vascular malformation of the brain stem served as subjects in this study. Thirty-three had angiographically visible arteriovenous malformations

Address for reprint requests: Raymond $\mathrm{N}$ Kjellberg, MD, Massachusetts General Hospital, 15 Parkman Street/ACC 324, Boston, MA 02114, USA.

Received 6 July 1987 and in revised form 10 June 1988. Accepted 14 August 1988
(AVAVMs) and 30 had angiographically occult vascular malformations (AOVMs). Patients with a venous abnormality on angiogram were placed in the AOVM group. All except five patients were treated with proton beam therapy. The number of patients with a brain stem AVAVM represent $3.6 \%$ of all the 927 patients with AVAVMs of the brain treated by proton beam therapy in our hospital from February 1965-May 1987 . The 25 patients with brain stem AOVM represent $54 \%$ of all the patients with AOVMs treated by proton beam therapy. Most of the patients with brain stem AOVM in this series were diagnosed and treated after the installation of an improved magnetic resonance machine in our hospital in 1985; hence the after therapy follow-up is relatively brief.

All patients had angiography and CT. Patients with AOVM underwent CT with $1.5 \mathrm{~mm}$ slices for reconstruction and then magnetic resonance imaging (MRI). MRI was performed using a superconductive whole-body system (Teslacon, Technicare Corp., Solon, Ohio) operating at 0.6T (25.4 MHz). $T_{1}$ and $T_{2}$-weighted spin echo sequences were used. Patients had axial and sagittal $T_{1}$ and $T_{2}$-weighted images.

\section{Case reports}

The following patient histories reveal some problems of diagnosis:

Patient 1 This 36 year old woman was in a motor vehicle accident 1 year prior to admission. About a week later she began to experience severe low back pain and an almost constant occipital throbbing headache. One month later, she noticed weakness, stiffness and sensory loss in all four extremities. These symptoms progressed over a period of months. Neurological examination on admission disclosed 
spastic quadriparesis, more pronounced on the right side. Sensory testing revealed decreased pain and touch on the left upper and lower extremities. Coordination and gait testing showed a mild bilateral incoordination, greater on the right. Cervical spine radiographs and noncontrast CT of the head were normal. Contrast CT showed an abnormal area of intense enhancement involving the pons (fig 1c). MRI showed an area of very low signal on $T_{1}$-weighted image in the pons (fig 1d). There were mixed high and low signals consistent with haemosiderin or flow effect on $\mathrm{T}_{2}$-weighted image (fig le). Left vertebral angiogram demonstrated an arteriovenous malformation (AVM) measuring $35 \times 27 \times$ $21 \mathrm{~mm}$ in the pons (fig $1 \mathrm{a}, \mathrm{b}$ ).

This patient presented with a progressive neurological deficit (PND) over several months. The possibility of a brain stem tumour was excluded by angiography and MRI, and the diagnosis of an AVM was confirmed.

Patient 2 This 43 year old woman was healthy until six years before admission, when she first experienced transient pulsating pain behind her left ear followed by rapid onset of numbness in the left side of the face, arm and leg associated with clumsiness and double vision. Most of her symptoms gradually disappeared, but she continued to have occasional double vision when lying on her left side. She was admitted for further investigation and treatment. Neurological examination showed partial right 6th nerve palsy, ocular dysmetria and flutter, saccadic lateral pursuit movement of the eyes and vertical nystagmus. There was also incoordination of the left arm and mild truncal ataxia. There was no weakness, sensory change or alteration of reflex in the extremities. CT revealed an area of hyperdensity in the midbrain and upper pons which was moderately augmented on enhancement (fig 2c). Vertebral angiogram showed no abnormality in the arterial phase. There was prominent veins within the region of the floor of the upper part of the fourth ventricle during the venous phase. There were also several foci of contrast puddling in the same region (fig $2 \mathrm{a}, \mathrm{b}$ ). MRI revealed a well defined lesion occupying the midbrain and pons with some tubular shaped areas of increased signal consistent with vessel thrombosis or old haemorrhage. There were also several tubular shaped areas of very low or absent signal representing flowing blood (fig $2 \mathrm{~d}, \mathrm{e}$ ). The $\mathrm{T}_{2}$ signal was

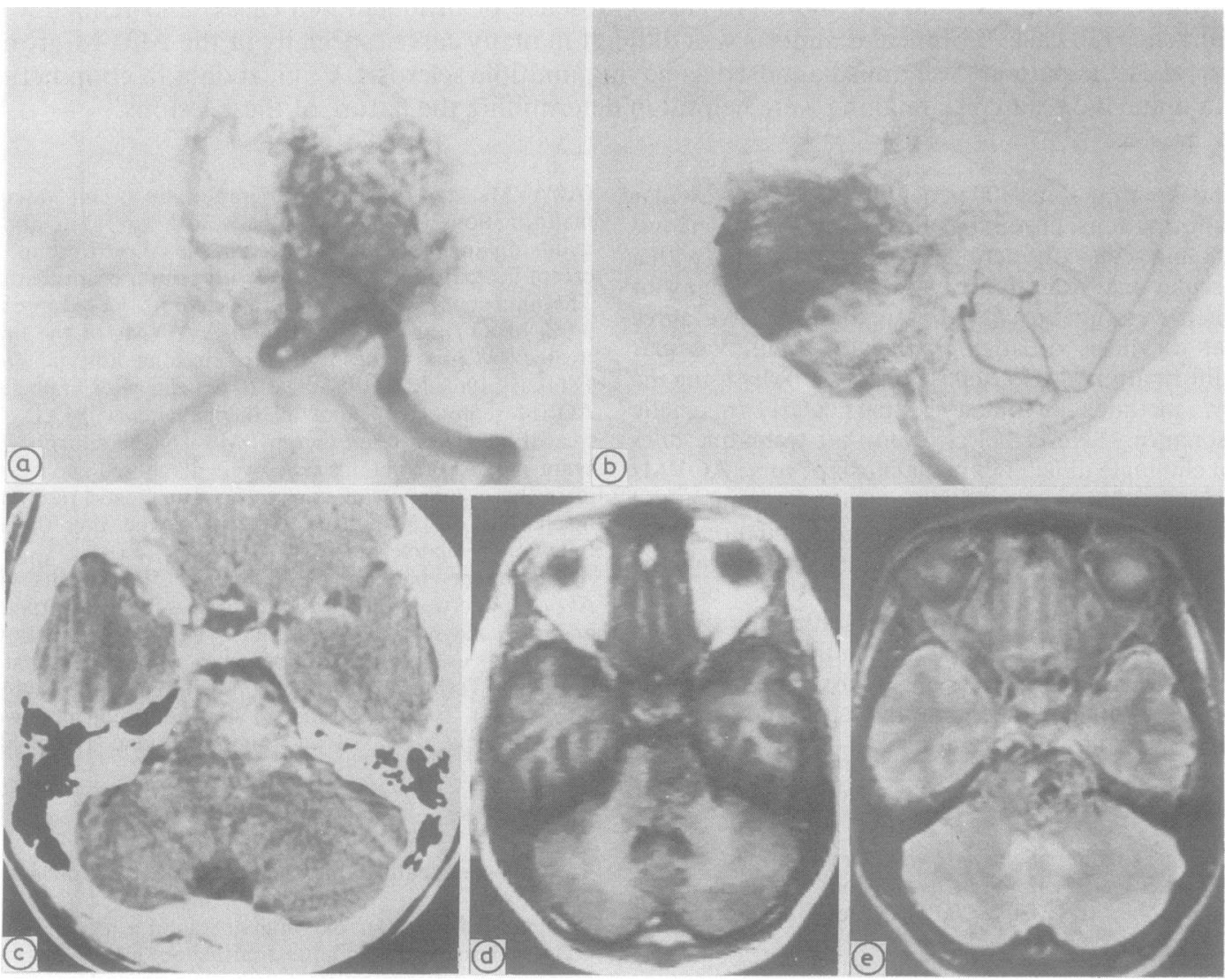

Fig 1 Patient 1. Anteroposterior (a) and lateral (b) views of left vertebral angiograms showing marked conglomeration of small vessels in the pons. CT scan with contrast shows a large area of intense enhancement involving the pons (c). MRI shows an area of decreased signal involving the pons with areas of very low signal in $T_{1}$-weighted image $(d)$. There are mixed high and low signals consistent with haemosiderin or flow effect in $T_{2}$-weighted image (e). 


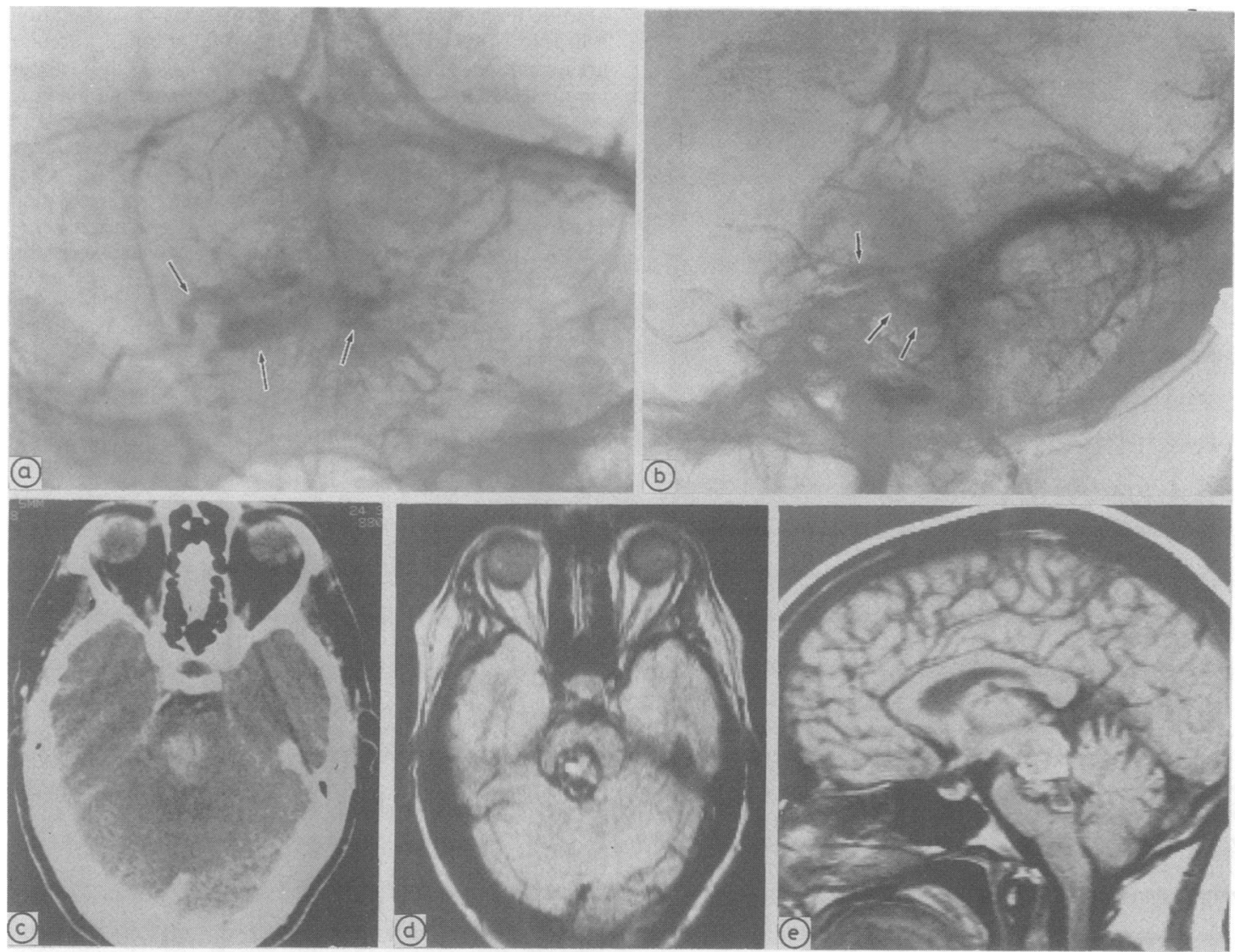

Fig 2 Patient 2. Anteroposterior (a) and lateral (b) views of left vertebral angiograms in the venous phase showing prominent veins (arrows) within the region of the floor of the fourth ventricle draining laterally towards the area of petrosal sinus. CT scan with contrast shows an area of hyperdensity in the upper pons (c). MRI demonstrates some tubular-shaped areas of increased signal consistent with thrombosis of vessels or old haemorrhage, and several tubular-shaped areas of very low or absent signal consistent with flowing blood in intermediate $T_{2}$-weighted axial image $(d)$, and in $T_{1}$-weighted sagittal image $(S E . T R=1000, T E=32)(e)$.

decreased at the margins of the lesion, a finding compatible with haemosiderin.

Prominent veins on angiogram, a well defined lesion with flow voids on MRI and absence of a mass effect indicated the existence of a vascular malformation. The neurological episode was probably caused by a small haemorrhage from the vascular malformation that left her with only minor deficits.

Patient 3 This 46 year old woman had onset of diplopia followed by numbness of the right half of her body two and a half years before admission. After CT was interpreted as normal, her illness was diagnosed as multiple sclerosis and she was given corticosteroid treatment. The symptoms cleared after one month. One and a half years later she had a similar but less severe episode which cleared after a few days. Seven months before admission a third episode occurred. Five months prior to admission repeated CT was again interpreted as being normal. Angiogram was normal; however, MRI showed a lesion in the brain stem. Neurological examination on admission revealed normal cranial nerves with the exception of primary position upbeat nystagmus, minimal paresis of upgaze, and right 4th nerve paresis. On sensory testing there was slightly decreased sensation to pain and temperature in the right upper extremity. The previous angiogram was reviewed and considered to be normal. High resolution CT with contrast showed the presence of a slightly hyperdense area in the left upper midbrain and the posteroinferior portion of the left thalamus. MRI showed a distinctly marginated abnormality in the posteromedial lower thalamus and the paramedian midbrain tegmentum on the left (fig 3 ).

The abnormality had the signal characteristics of a haemorrhage. Here the patient had three minor attacks of neurological disease suggestive of a lesion in the brain stem. The haemorrhage had been too small to be seen on nonenhanced CT. Only when MRI disclosed evidence of 


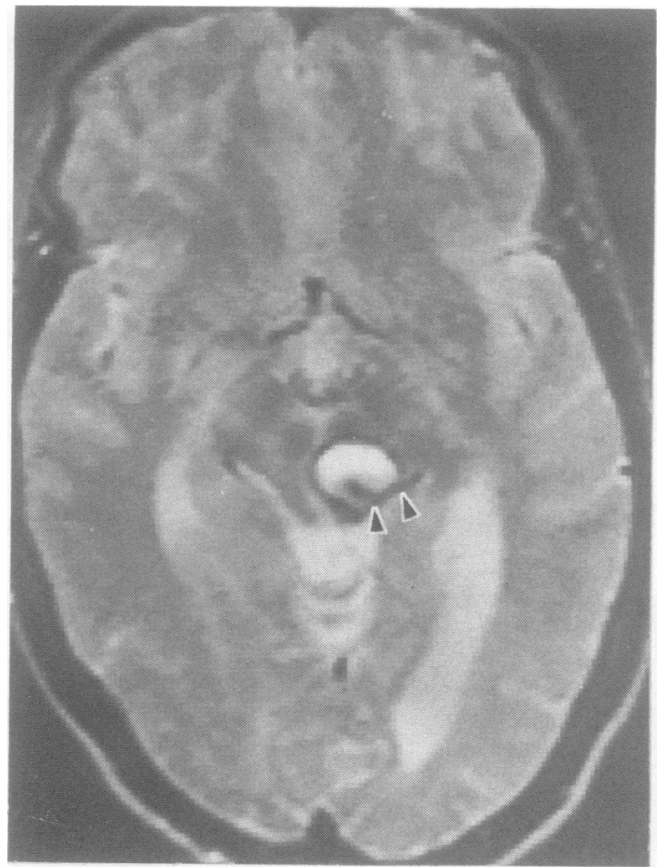

Fig 3 Patient 3. MRI shows a distinctly marginated increased signal in $T_{2}$-weighted image. Curvilinear structure of decreased signal (arrows) from the posterolateral border of the lesion suggestive of a flow void from an abnormal vein. Decreased signal is compatible with haemosiderin at the margin and within the lesion.

haemorrhage and the absence of a tumour mass in a lesion at least two and a half years old, was its nature clarified. Recurrence of episodes and flow void on MRI suggested the existence of a vascular malformation.

Patient 4 This 40 year old man was well until 12 years ago when, while exercising, he noted acute onset of diplopia, vertigo, numbness and tightness over the right face and body. Clumsiness of the right hand and slurred speech developed a few days after onset. The symptoms lasted five weeks and then gradually receded, though not completely. The illness was diagnosed as multiple sclerosis. Similar episodes recurred five and seven years later, lasting one and several weeks respectively. The residual symptoms were more pronounced after each episode. An angiogram six months before admission disclosed no abnormality. Neurological examination on admission showed normal cranial nerves, except for bilateral endgaze nystagmus and nonparalytic esophoria of the right eye. There was minimal decrease of pain and slight dysmetria in the right arm. CT showed a hyperdense area after injection of contrast. MRI revealed a lesion with the signal characteristics of an old blood clot. Bilateral vertebral angiogram was repeated and was again normal. He was given proton beam therapy for his pontine vascular malformation.

Three months after therapy there was progressive neurological deterioration and he died eight months later. Postmortem examination revealed haemorrhage in the left pontine tegmentum surrounded by an area of coagulation necrosis. Microscopic sections disclosed a small vascular malformation adjacent to the haemorrhage (fig 4 upper picture). There were several dilated blood vessels, mainly small veins, whose walls were replaced by collagen fibres and hyalinised tissue. No definite elastic laminae were seen in any of these vessels; a few were markedly ectatic. There was a clot inside one extremely thin-walled vessel measuring $2 \times 3 \mathrm{~mm}$. There was focal interruption of the wall in one of the serial sections, fresh haemorrhage, and a layer of haemosiderin laden macrophages around the blood vessel (fig 4 lower picture) consistent with recurrent haemorrhage. There was also gliotic brain tissue among the blood vessels. A capillary venous malformation was the final diagnosis.

A vascular malformation was the cause of the haemorrhage which recurred over a period of 12 years following proton beam therapy and ultimately took the patient's life.

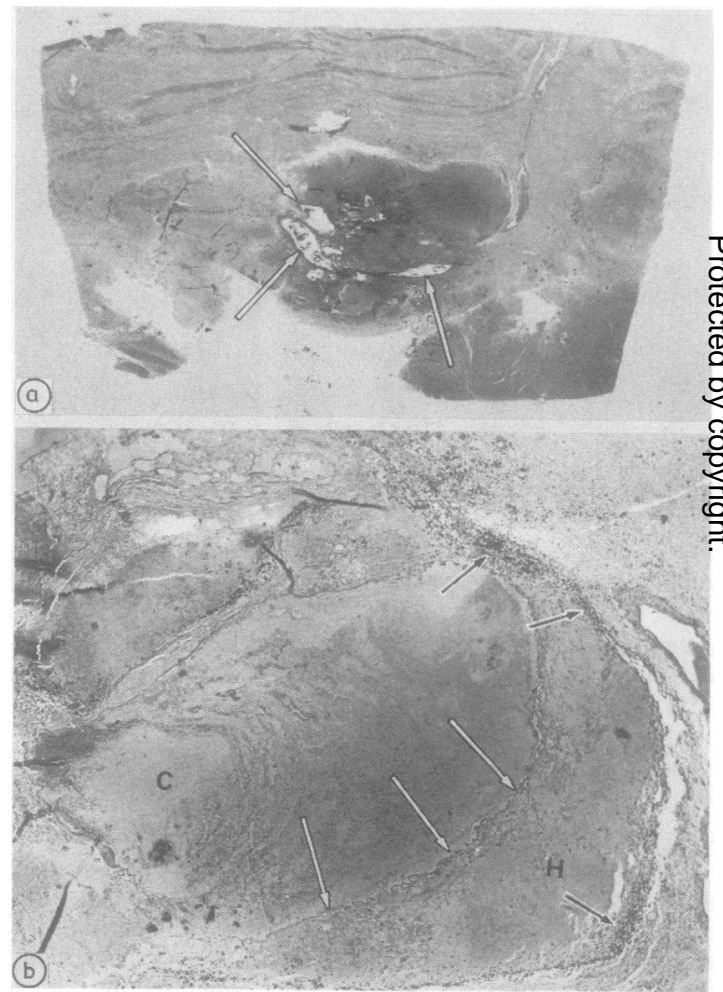

Fig 4 Patient 4. Autopsy. Section of posterior half of pons (upper picture) shows a haemorrhage in the median and left paramedian pontine tegmentum. Some prominent blood vessels (white arrows) in the area of haemorrhage.

van Gieson $\times 2$. Microscopic examination (lower picture) shows a markedly ectatic blood vessel, $2 \times 3 \mathrm{~mm}$, with very thin wall (white arrows). A clot $(C)$ is inside this blood vessel. Haemorrhage $(H)$ and haemosiderin laden microphages (black arrows) around the blood vessels. No elastic laminae in the wall of blood vessels. van Gieson $\times 22$. 


\section{Results}

Comparison of the two groups of vascular malformation In 33 patients with AVAVM 18 were male and 15 female. In 30 patients with AOVM 11 were male and 19 female. There were three main types of symptom presentation: progressive neurological deficit (PND), stroke due to frank haemorrhage, and indistinct but probable haemorrhage. Patients with AVAVM came to medical attention because of either PND (12/ $33=36 \%$ ) or stroke with frank haemorrage $(20 / 33=61 \%)$. In over $70 \%$ the age at onset of the first symptom was between 20 and 39 years (table 1). Patients with AOVM presented with stroke due to frank haemorrhage $(4 / 30=13 \%)$, indistinct haemorrhage $(25 / 30=83 \%)$ and PND $(1 / 30=7 \%)$ as the first symptom. In over $70 \%$ the age at onset of haemorrhage was between 10 and 39 years.

\section{Clinical course}

AVAVM: Patients with PND followed an irregularly progressive course without a period of improvement, except for one patient who had worsening of PND during pregnancy and improved after delivery. One patient who presented with PND as the first symptom later suffered haemorrhage. Eleven patients with PND showed progression of symptoms over the course of more than a year. Seventeen patients had had a frank subarachnoid or intraventricular haemorrhage while three patients had intraparenchymal haemorrhage. Fourteen patients had only one haemorrhage, seven patients two or more (table 2). Successive haemorrhages occurred within a 6 month interval in nine of the 17 with recurrent haemorrhages. Two patients who had haemorrhage as the first symptom later exhibited PND. One patient presented with only trigeminal neuralgia.

AOVM: Five patients of 25 who had an indistinct haemorrhage as the first symptom suffered frank haemorrhage in subsequent episodes. Twenty patients had recurrent episodes of haemorrhage; eight had two haemorrhages, seven had three, four had four, and one had six (table 2). The interval between episodes varied widely with PND as the first symptom. There was one patient who presented with haemorrhage later than PND.

The presentation pattern of haemorrhage was remarkably variable in the AOVM group. In many patients it was not clearly apoplectic. The symptoms evolved one after another or progressed in intensity for a period of several days. A detailed history of onset could be obtained in 58 of 67 episodes. Evolution of symptoms was within a day in 27; 2 days to within a week in 23; more than a week to one month in eight, and was undetermined in nine episodes (table 3 ). If undetermined episodes were to be excluded, evolution
Table 1 Age at onset of the first symptom

\begin{tabular}{cccc}
\hline Age (yr) & AVAVM (PND) & AVAVM (Haem) & AOVM (Haem)* \\
\hline $0-9$ & 0 & 2 & 2 \\
$10-19$ & 1 & 2 & 6 \\
$20-29$ & 4 & 4 & 8 \\
$30-39$ & 5 & 10 & 7 \\
$40-49$ & 2 & 1 & 3 \\
$50-59$ & 0 & 1 & 3 \\
$60-69$ & 0 & 0 & 0 \\
Total & 12 & 20 & 29
\end{tabular}

*: Patients with angiographically visible arteriovenous malformation (AVAVM) presenting with progressive neurological deficit (PND), patients with AVAVM presenting with haemorrhage (Haem), and patients with angiographically occult vascular malformation (AOVM) presenting with haemorrhage.

Table 2 Frequency of haemorrhage

\begin{tabular}{lcc}
\hline No. of haemorrhages & AVAVM & AOVM* \\
\hline 0 & 12 & 1 \\
1 & 14 & 9 \\
2 & 3 & 8 \\
3 & 2 & 7 \\
4 & 0 & 4 \\
5 & 1 & 0 \\
$5+$ & 1 & 1 \\
Total & 33 & 30 \\
\hline
\end{tabular}

*: Patients with angiographically visible arteriovenous malformation (AVAVM) and patients with angiographically occult vascular malformation (AOVM).

Table 3 Mode of onset in patients with angiographically occult vascular malformation ( $A O V M$ )

\begin{tabular}{lrcr}
\hline & \multicolumn{3}{l}{ Venous abnormality on angiogram } \\
\cline { 2 - 4 } & $(+)$ & $(-)$ & Total $(\%)$ \\
\hline Evolution of symptoms & 11 & 16 & $27(40 \%)$ \\
$>\quad 1$ day & 5 & 18 & $23(34 \%)$ \\
$2-7$ days & 3 & 5 & $8(22 \%)$ \\
8-30 days & 1 & 8 & $9(13 \%)$ \\
undetermined & 20 & 47 & 67 \\
Total No. episodes & & & \\
\hline
\end{tabular}

of symptoms in a period of 2 days or more occurred in 31 out of 58 . Symptoms and neurological signs were slight in some episodes and severe in others. In order to analyse patterns of recurrence we divided the episodes into minor and major types, according to the severity of symptoms. Only minor episodes were noted in 13 out of 29 patients. Six patients had a minor episode first and later experienced a major one. When the mode of onset and patterns of recurrence were studied in relation to the presence or absence of venous abnormality on angiogram, the mode of onset did not differ significantly in the two groups. Recurrent minor episodes were frequently noted in patients with a normal angiogram. Five out of seven patients with a venous abnormality had experienced at least one major episode. 
Symptomatology

Frequency of headache per episode was $93 \%$ in the AVAVM group, while it was recorded in only $25 \%$ in the AOVM group. Four out of 21 of patients with AVAVM became comatose at the time of haemorrhage. In none of those with AOVM did coma occur although it was attended by an initial disturbance of consciousness, confusion or drowsiness in four patients. Frequent symptoms in the AOVM group were diplopia (72\%) and unilateral sensory disturbances (55\%). Hemiparesis, clumsiness, diplopia, hearing difficulty and unsteady gait were frequent in both groups (table 4). Six patients with AVAVM and two with AOVM had frequent headaches. Three of them (two AVAVM, one AOVM) had a migraine syndrome. One patient with AVAVM experienced a single seizure just after the haemorrhage. None of the patients had subjective bruit. A cranial bruit was detected in only one patient with AVAVM.

In contrast to this series in our large group of 894 patients with AVAVMs of other parts of the brain, 403 presented with haemorrhage, 214 with seizure, 177 with headache and 80 with PND as the first symptom.

\section{Neurological findings}

As would be expected from the location of the lesions, nystagmus, neurosensory deafness, dysarthria, hemiparesis, hemisensory disturbance, limb ataxia and truncal ataxia were frequent in both groups of patients. Various neuro-opthalmological findings were particularly prominent in patients with AOVM and were useful in assessing the level of brain stem involvement. Twenty-two patients with AVAVM had

Table 4 Frequency of symptoms

\begin{tabular}{lllc}
\hline Symptom & $\begin{array}{l}\text { AVAVM } \\
(\text { PND })\end{array}$ & $\begin{array}{l}\text { AVAVM } \\
\text { (Haem) }\end{array}$ & $\begin{array}{c}\text { AOVM* } \\
\text { (Haem) }\end{array}$ \\
\hline Headache† & $0 /$ & $37 / 40$ & $17 / 67$ \\
Nausea, vomiting & $0 /$ & $15 / 21$ & $7 / 29$ \\
Coma & $0 /$ & $4 / 21$ & $0 /$ \\
Diplopia & $4 / 14$ & $8 / 21$ & $21 / 29$ \\
Facial weakness & $1 / 14$ & $4 / 21$ & $4 / 29$ \\
Hearing difficulty & $2 / 14$ & $4 / 21$ & $5 / 29$ \\
Vertigo & $0 /$ & $0 /$ & $4 / 29$ \\
Dizziness & $0 /$ & $1 / 21$ & $8 / 29$ \\
Dysarthria & $1 / 14$ & $1 / 21$ & $5 / 29$ \\
Swallowing difficulty & $2 / 14$ & $1 / 21$ & $1 / 29$ \\
Hemiparesis & $6 / 14$ & $8 / 21$ & $10 / 29$ \\
Unilateral sensory disturbance & $1 / 14$ & $2 / 21$ & $16 / 29$ \\
Clumsiness of limbs & $6 / 14$ & $5 / 21$ & $5 / 29$ \\
Unsteady gait & $5 / 14$ & $2 / 21$ & $10 / 29$ \\
Resting tremor & $2 / 14$ & $1 / 21$ & $4 / 29$ \\
Slurring of speech & $2 / 14$ & $1 / 21$ & $6 / 29$ \\
\hline
\end{tabular}

*: Patients with angiographically visible arteriovenous malformation (AVAVM) presenting with progressive neurological deficit (PND), patients with AVAVM presenting with haemorrhage (Haem), and patients with angiographically occult vascular malformation (AOVM) presenting with haemorrhage.

$\dagger:$ Frequency per episode. Frequency per person in other symptoms. major neurological deficits but only two of them were totally incapacitated. Patients with AOVM had less severe disabilities than patients with AVAVM.

\section{Radiographic features: Angiography}

$A V A V M$ : vertebral angiograms characteristically revealed closely packed small vessels in the early arterial phase and dilated draining veins in the midarterial phase consistent with the diagnosis of AVM. The size of AVMs (maximum dimension in angiogram divided by the magnification of the film) averaged $42.0 \mathrm{~mm}$ in patients with PND and $35.00 \mathrm{~mm}$ in patients with haemorrhage.

AOVM: Angiograms showed a venous abnormality in only eight of 30 patients and no abnormality whatsoever in the other 22. Six patients had a typical or atypical caput medusae pattern, that is, a network of medullary veins converging centrally into a single larger venous channel issuing into the venous system. Two patients had a prominent vein with foci of contrast puddling. In three out of six patients with caput medusae pattern, however, there was considerable distance between the location of a venous abnormality and that of the lesion shown by CT and MRI.

\section{Computed tomography}

$A V A V M: C T$ with contrast was superior to $\mathrm{CT}$ withor contrast in showing a definite confluent pattern of enchancement and thus revealed the location of the $T$ AVM in all cases. The pons was involved in 24 过 patients, midbrain in 18 patients, and medulla in ong patient. There was extension to the cerebellum in 1

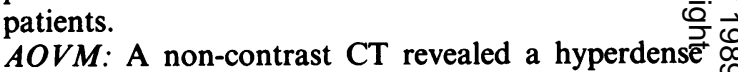
lesion in most patients. It should be emphasised that a hyperdense area of this type can be due to a blood clot or calcification. Distinct calcification was shown in seven patients and fresh haemorrhage in five. A hypodense area, consistent with a resolving blood clot, was also seen in a few patients. Enhancement with contrast was slight to moderate in 18 out of 22 patients; in four there was no enhancement or only a questionable positivity even with high quality images. The border of hyperdense or enhanced lesion was usually obscure except for lesions with fresh haemorrhage or prominent calcifications.

\section{$M R I$}

AVAVM: Only two patients in this group had MRI. The large vascular components of AVM usually appeared as a lattice work of signal void spaces, highly 0 contrasted with the surrounding brain tissue on both $\mathrm{T}_{1}$ - and $\mathrm{T}_{2}$-weighted images.

AOVM: Twenty-eight of the patients in this group had MRI. Here the MRI was particularly useful in N detecting old haemorrhage which had increased $N$ 
signal characteristics of haemoglobin derivatives (methemoglobin) combined with a decreased signal compatible with haemosiderin. In most of the patients haemorrhage had the characteristic concentric configuration with methaemoglobin in the central portion and haemosiderin in the peripheral. Calcification, characterised by profoundly reduced signal intensity was distinguished from haemorrhage by their opposite effects on signal intensity. Twenty-five out of the 27 patients who had neurological episodes and underwent MRI showed signal characteristics of methaemoglobin and haemosiderin.

The location and size of haemorrhage demonstrated by MRI was of particular interest in patients with AOVM. Haemorrhage was shown in the pontine tegmentum (5/29) and other areas (5/29). Most of the haemorrhage $(24 / 29)$ was in the paramedian region. All 29 patients who presented with episodic manifestations had intraparenchymal blood clot or haemosiderin demonstrated by CT or MRI. Three patients with a venous abnormality had subarachnoid or intraventricular haemorrhage in addition to intraparenchymal haemorrhage. The maximum diameter of haemorrhage on axial MRI was smaller than $20 \mathrm{~mm}$ in 14 patients; between 20 to $30 \mathrm{~mm}$ in eight patients. The size of haemorrhage tended to correspond to severity of symptoms. Patients with minor episodes had haemorrhage of 0-20 mm, while patients with major episodes had haemorrhage of 10 $30 \mathrm{~mm}$. No significant difference in size or location was noted between AOVM with a venous abnormality and AOVM with a normal angiogram.

\section{Pathological diagnosis}

Three patients with AOVM underwent craniotomy and biopsy, and one patient with AOVM had a stereotactic biopsy in another hospital. Pathological study showed a vascular malformation in two patients and blood clot with altered brain tissue in another two patients. One patient (Patient 5) died and at necropsy there was a capillary venous malformation in the pontine tegmentum.

\section{Discussion}

The main point arising from this study of a large series of brain stem malformations is that nearly half of them were not angiographically visible. The existence of small vascular malformations not demonstrable by angiography is, of course, not a novel observation and there have been many reports of such conditions. ${ }^{2-46-14}$ This large series, however, affords a broad view of their unique clinical characteristics and an opportunity to compare them with a group of AVAVMs.

Another of the principal points to be made is the frequency of vascular malformations in the brain stem. Brain stem AVAVMs represent only $3.6 \%$ of all AVAVMs of the brain in our series and only 2 to $4.8 \%$ in the other major reported clinical series. ${ }^{15-18}$ Moreover, the AOVMs in our series showed a marked predominance $(54 \%)$ for the brain stem. This may of course be due to the special referral pattern of patients. Probably important is the neuroanatomy of the brain stem, where its small size and small intrinsic vasculature would favour small vascular malformations and where even a small haemorrhage would be expected to evoke conspicuous neurological deficits. Whatever the reasons, the distribution pattern of AOVM predominates in the brain stem. McCormick et $a l^{19}$ have made a similar observation that the distribution of small vascular malformations was almost equal between supra and infratentorial sites in necropsy cases, whereas a striking majority of large AVMs are supratentorial.

\section{Notable clinical attributes}

The qualities of the haemorrhage syndrome deserve comment. Haemorrhage, which was the most frequent presentation with brain stem AVAVM, was usually dramatic and grave. It left many patients with major neurological deficits. Recurrence was frequent and tended to occur within 6 months. Small haemorrhages may have been the cause of PND in some patients. However, gradual progression of symptoms over a period of a year could also be explained by mechanical compression or ischaemia due to a slowly enlarging AVM.

In our group of AOVM, frank subarachnoid haemorrhage was rare (only three of 30 ). Proven parenchymal haemorrhage, which is the principal mechanism of symptom production in AOVM, was seldom apoplectic; headache was absent $(75 \%)$ and the mode of onset was neither sudden nor acute in many episodes. Combined with the high frequency of recurrence $(69 \%)$, these characteristics constituted a unique clinical syndrome quite atypical of intracranial haemorrhage.

There are several other reports also suggesting that "fluctuating or intermittently progressive symptoms of long duration" are characteristic clinical presentations of brain stem vascular malformations. ${ }^{46} 111420-22$ Presumably these syndromes are caused by haemorrhages which resolved and recurred. Only by CT and MRI can the haemorrhagic nature of the lesion be ascertained. In many cases it was the succession of these neurological episodes, all within the same general locality of the brain stem, that gave rise to the question of demyelinative, inflammatory or neoplastic lesion.

Of assistance in the differentiation of AOVM from demyelinative disease is the single locale of disease; multiplicity of lesions is an essential criterion for multiple sclerosis. Of course, that may not be true at the time of the first attack. Also, there may in some cases be multiple small vascular malformations which will then create further diagnostic difficulties. MRI 
demonstration of blood flow is helpful. Other types of haemorrhage must be excluded. Most patients with primary pontine haemorrhage owing to hypertension exhibit a more rapid and devastating neurological deterioration. ${ }^{23}$ If small, the hypertensive haemorrhage is usually localised in the tegmentum and difficult to differentiate from that of AOVM; if it is large, it involves the entire pons and usually results in death.

A progressive neurological deficit (PND) is difficult to differentiate from haemorrhage by clinical features alone in patients with AOVM. Our definition of a PND is a syndrome which continues to progress for more than a month and shows no significant improvement after that. Progressive neurological deficit occurred in two out of 30 AOVMs and in one of them it was preceded by four episodes of indistinct haemorrhage. In these patients with PND a brain stem glioma, a parasitic infection, or some other inflammatory process needed to be differentiated. Here, the most helpful points in diagnosing the vascular malformation were lack of obvious mass effect in a long standing lesion and presence of recent haemorrhage. Only in the malignant gliomas and a few metastatic tumours are haemorrhages from abnormal vessels likely to develop and their clinical course is usually rapidly progressive. Angiography, which has been the most reliable radiographic means in diagnosing AVMs, may be difficult to interpret. A venous abnormality on angiography representing a venous angioma ${ }^{24}$ can be no more than a coincidental finding ${ }^{25}$ or may only indicate the presence of AOVM in an adjacent area; Roverson et $a P^{26}$ and Diamond et $a P^{7}$ reported three cases in which a venous abnormality was seen in the cerebellum on angiogram while a vascular malformation with haemorrhage was found in the pons. Also, in some of our patients with AOVM, there was a considerable distance between the location of a venous abnormality and that of a lesion shown by both CT and MRI. Three patients with a venous abnormality had a clinical subarachnoid haemorrhage, which did not occur in any of the patients with a normal angiogram. While this may suggest a more potentially devastating complication of AOVM with venous abnormality, the number is too small to be of significance.

The interpretation of CT where an isodense or slightly hyperdense focus enhanced to a minimal or moderate degree with contrast was also difficult in some instances. The problem confronting the radiologist in the differential diagnoses of AOVM on CT was that of distinguishing it from a low grade astrocytoma, oliogodendroglioma or granulomatous lesion. Our experience leads us to believe that MRI is optimal for identifying the characteristic and specific signal alterations of old haematomas, ${ }^{132829}$ which can not be detected by CT once they have lost density with time and are obscured by other structures in the posterior fossa. Blood clot may be the only finding in
AOVM patients. Often it had a concentric configuration with methemoglobin in the central portion and haemosiderin in the periphery. Gomori et al speculated that the rounded deposits seen in AOVM might be consistent with recurrent small haemorrhages and nonresorbable nidus of vascular malformation. This round concentric pattern is not specific for AOVM; nevertheless, it is the most characteristic pattern of haemorrhage from AOVM.

\section{Nature of the lesion}

Relatively slow progression of symptoms, which is the characteristic neurological presentation of haemorrhage from AOVM, is presumably due to the oozing of blood under low pressure from small vessels. In the clinical series of AOVMs of the brain that have been verified histologically, small AVMs with thrombosis of some of the vessels have been reported. ${ }^{39101213}$ Several reported cases with a histologically verified vascular malformation of the brain stem were classified as capillary telangiectases, venous angiomas or cavernous angiomas. ${ }^{91420212627}$ None of them were angiographically evident. They were as frequent as AVMs that were not visible on angiogram. However the number of cases is too small to afford a cleas picture of their morphology. In one of the fevo pathology studies, McCormick et al ${ }^{\beta 0}$ compared vas cular malformations of the posterior fossa with those $\vec{T}$ in the cerebrum. Those in the brain stem were $\mathbb{D}$ subdivided into capillary telangiectases $(44 \%)$, AVM 8 $(25 \%)$, venous angiomas $(15 \%)$ and cavernous angiomas $(14 \%)$ while in the other parts of the brai most $(62 \%)$ were AVMs. In our experience th histological classification is rather arbitrary and frequently difficult to apply to small vascular malformations. Certainly any of these types can cause small, recurrent haemorrhages.

\section{Natural course of vascular malformations}

Crawford $e t a^{\beta^{1}}$ recently reported on the natural history of all AVMs of the brain. The analysis disclosed a $42 \%$ risk of haemorrhage, $29 \%$ risk of death and $27 \%$ risk of having a neurological handicap by 20 years. Neurological handicap or risk of death in brain stem AVAVMs appears higher than in AVAVMs of other regions of the brain because the haemorrhages involve so many vital structures crow- 3 ded into a small region. Many of our patients with brain stem AVAVM actually had major neurological disabilities as a result of PND and/or devastating haemorrhage. Fults $e t a^{22}$ reported the follow-up 을 results of posterior fossa AVAVMs; the mortality was $\frac{D}{O}$ $66.7 \%$ with the first haemorrhage, and in survivors there were, as a rule, recurrent haemorrhages and most $N$ of these were fatal.

The natural history of AOVM has not yet been studied. Some case reports of those in the brain stem $\mathrm{\omega}$ appear to describe a variable outcome; some pursuing 
a rapidly deteriorating course and others surviving for a long time with a fluctuating course. ${ }^{4571120-22}$ Fortyfive per cent of our patients with AOVM had only minor episodes and were doing well with minor deficits. On the other hand $21 \%$ of patients had a minor episode initially and then subsequently suffered major episodes. Although the grade of neurological disability in our patients with AOVM was not as severe as in patients with AVAVM, almost half of them had some degree of neurological deficit. In consideration of these, AOVMs which once cause haemorrhage can be regarded as potentially disabling.

Neurosurgical removal or coagulation of AVAVMs in the brain stem rarely could be considered. The surgical mortality rates are high (up to $30 \%$ ) and complete removal even of small superficial AVAVMs is very difficult. ${ }^{151618}$ The surgical evacuation of blood clots and surgical resection of AOVM have been recommended in some cases, ${ }^{73}$ but are rarely indicated because the typically small clots will absorb naturally and surgical morbidity carries an unacceptable risk. Conventional radiation therapy has been reported to be effective in obliterating cavernous angiomas in the brain stem diagnosed with CT. ${ }^{34}$ Details of stereotactic Bragg peak proton beam therapy have not been discussed here because so far the follow-up period is too short for the results to be significant.

We thank Dr Kenneth R Davis and Dr Tessa HedleyWhyte for consultation; Ms Kristen Hughes, Ms Vicky Martin and Ms Rose-Marie Moffet for helping in preparing the manuscript; and Dr Wilson T Asfora and Dr Antonio A F DeSalles for advice and support.

\section{References}

1 Kucharczyk W, Lemme-Pleghos L, Uske A, Brant-Zawadzki M, Dooms G, Norman D. Intracranial vascular malformations: MR and CT imaging. Radiology 1985;156:383-9.

2 Gomori JM, Grossman RI, Goldberg HI, Hackney DB, Zimmerman RA, Bilaniuk LT. Occult cerebral vascular malformations: high-field MR imaging. Radiology 1986;158:707-13.

3 New PFJ, Ojemann RG, Davis KR et al. MR and CT of occult vascular malformations of the brain. AJNR 1986;7:771-9.

4 Britt RH, Connor WS, Enzmann DR. Occult arteriovenous malformation of the brainstem simulating multiple sclerosis. Neurology 1981;31:901-3.

5 Sadeh M, Shacked I, Rappaport ZH, Tadmor R. Surgical extirpation of a venous angioma of the medulla oblongata simulating multiple sclerosis. Surg Neurol 1982;17:334-7.

6 Yeates A, Enzmann D. Cryptic vascular malformations involving the brain stem. Radiology 1983;146:71-5.

7 Veerapen RJ, Sbeih IA, O'Laoire SA. Surgical treatment of cryptic AVM's and associated hematoma in the brain stem and spinal cord. J Neurosurg 1986;65:188-93.

8 Bell BA, Kendall BE, Symon L. Angiographically occult arteriovenous malformations of the brain. J Neurol Neurosurg Psychiatry 1978;41:1057-64.

9 Becker DH, Townsend JJ, Kramer RA, Newton TH. Occult cerebrovascular malformations. Brain 1979;102:249-87.

10 Shuey HM, Day AL, Quisling RG, Sypert GW. Angiographically cryptic cerebrovascular malformations. Neurosurgery 1979; 5:476-9.

11 Albright AL, Byrd RP, Harrison ML. Angiographically cryptic AVM presenting as a pontine tumor. Case report. J Neurosurg 1980;53:846-8.

12 Cohen HCM, Tucker WS, Humphreys RP, Perrin RJ. Angiographically cryptic histologically verified cerebrovascular malformations. Neurosurgery 1982;10:704-14.

13 Wakai S, Ueda Y, Inoh S, Nagai M. Angiographically occult angiomas: a report of thirteen cases with analysis of the cases documented in the literature. Neurosurgery 1985;17:549-56.

14 El-Gohary EM, Tomita T, Gutierrez FA, McLone DG. Angiographically occult malformations in childhood. Neurosurgery 1987;20:759-66.

15 Batjer H, Samson D. Arteriovenous malformations of the posterior fossa. Clinical presentation, diagnostic evaluation, and surgical treatment. $J$ Neurosurg 1986;64:849-56.

16 Drake CG, Friedman AH, Peerless S. Posterior fossa arteriovenous malformations. $J$ Neurosurg 1986;64:1-10.

17 Perret G, Nishioka $H$. Report on the cooperative study of intracranial aneurysms and subarachnoid hemorrhage. Section VI. Arteriovenous malformations. J Neurosurg 1966;25:467-90.

18 Solomon RA, Stein BM. Management of arteriovenous malformations of the brain stem. $J$ Neurosurg 1966;24:865-75.

19 McCormick WF, Nobzinger JD. "Cryptic" vascular malformations of the central nervous system. J Neurosurg 1966;24: 865-75.

20 Teilman K. Hemangiomas of the pons. Arch Neurol Psychiatry 1953;69:208-23.

21 Stahl SM, Johnson KP, Malamud N. The clinical and pathological spectrum of brain-stem vascular malformations. Long-term course simulates multiple sclerosis. Arch Neurol 1980;37:25-9.

22 DeJong RN, Hicks SP. Vascular malformation of the brainstem: report of a case with long duration and fluctuating course. Neurology 1980;30:995-7.

23 Weisberg LA. Primary pontine hemorrhage: clinical and computed tomographic correlations. J Neurol Neurosurg Psychiatry 1986;49:346-52.

24 Wendling LR, Moore (Jr) JS, Kieffer SA, Goldberg HI, Latchaw RE. Intracerebral venous angioma. Radiology 1976;119:141-7.

25 Saito Y, Kobayaski N. Cerebral venous angiomas. Radiology 1981;139:87-94.

26 Roberson GM, Kase CS, Wolpow ER. Telangiectases and cavernous angiomas of the brain stem: "cryptic" vascular malformation. Neuroradiology 1974;8:83-9.

27 Diamond C, Torvik A, Amundsen P. Angiographic diagnosis of telangiectases with cavernous angioma of the posterior fossa: report of two cases. Acta Radiol (Stockh) 1976;17:281-8.

28 Gomori JM, Grossman RI, Goldberg HI, Zimmerman RA, Bilaniuk LT. Intracranial hematomas: imaging by high-field MR. Radiology 1985;157:87-93.

29 Edelman RR, Johnson K, Buxton R et al. MR of hemorrhages: a new approach. $A J N R$ 1986;7:751-6.

30 McCormick WF, Hardman JM, Lieutenant MC, Boulter TR. Vascular malformations ("Angiomas") of the brain with special reference to those occurring in the posterior fossa. $J$ Neurosurg 1968;28:241-51.

31 Crawford PM, West CR, Chadwick DW, Shaw MDM. Arteriovenous malformations of the brain: natural history in unoperated patients. J Neurol Neurosurg Psychiatry 1986;49: 1-10.

32 Fults D, Kelly (Jr) DL. Natural history of arteriovenous malformations of the brain: a clinical study. Neurosurgery 1984;15:658-62.

33 Scott BB, Seeger JF, Schneider RC. Successful evacuation of a pontine hematoma secondary to rupture of a pathologically diagnosed "cryptic" vascular malformation. Case report. J Neurosurg 1973;39:104-8.

34 Yamasaki T, Handa H, Yamashita J et al. Intracranial and orbital cavernous angiomas. A review of 30 cases. J Neurosurg 1986;64:197-208. 